\title{
EFECTO DE VARIOS NIVELES DE ENERGÍA DIGESTIBLE Y PROTEÍNA EN LA DIETA SOBRE EL CRECIMIENTO DE GAMITANA (Colossoma macropomum) CUVIER 1818
}

\author{
Effect of Various Levels of Digestible Energy and Protein in the Diet on \\ the Growth of "Gamitana" (Colossoma maCropomum) Cuvier 1818
}

Félix Walter Gutiérrez A. ${ }^{1,2}$, Javier Zaldívar R. ${ }^{1}$ y Guadalupe Contreras S. ${ }^{1}$

\section{Resumien}

\begin{abstract}
Se llevó a cabo un experimento factorial para examinar el efecto de la interacción de dos niveles de proteína ( 25 y $35 \%$ ) y tres niveles de energía digestible $(2.3,2.5$ y $2.7 \mathrm{kcal} /$ g de alimento) en la dieta sobre la ganancia de peso (GP), conversión alimenticia (CA), energía retenida (ER) y el cociente de eficiencia proteica (REP) en la gamitana (Colossoma macropomum). En la composición de las dietas se empleó harina de anchoveta y harina de torta de soya como fuentes de proteína y maíz amarillo duro, subproducto de trigo y subproducto de cervecería como fuentes de energía. Se encontraron interacciones altamente significativas $(\mathrm{p}<0.01)$ entre la energía digestible y la proteína. En las dietas con $25 \%$ de proteína, el incremento del nivel de energía digestible mejoró significativamente la GP, CA, ER y la REP, siendo la mejor respuesta con $2.7 \mathrm{kcal} / \mathrm{g}$. Sin embargo, para el nivel de $35 \%$ de proteína, solo el nivel de $2.7 \mathrm{kcal} / \mathrm{g}$ influyó significativamente sobre el comportamiento productivo de la gamitana. Se concluye que la gamitana obtuvo mejor rendimiento cuando fue alimentada con una dieta de $2.7 \mathrm{kcal} / \mathrm{g}$ de energía digestible y $25 \%$ de proteína.
\end{abstract}

Palabras clave: energía digestible, proteína, requerimientos nutricionales, Colossoma

\section{Abstract}

\begin{abstract}
A factorial experiment was conducted to evaluate the effect of diets with two levels of protein (25 and 35\%) and three levels of digestible energy $(2.3,2.5$, and $2.7 \mathrm{kcal} / \mathrm{g}$ ) on the "gamitana" Colossoma macropomum in terms of body weight gain (WG), food conversion (FC), retained energy (RE), and protein efficiency ratio (PER). Diets contained anchovy and soybean meal as protein sources, and yellow corn, wheat bran, and brewery bran as energy sources. There were highly significant interactions $(p<0.01)$ between protein concentration and digestible energy on the "gamitana" growth. In diets with $25 \%$
\end{abstract}

${ }^{1}$ Estación Experimental del Centro de Investigación IVITA-Pucallpa, Facultad de Medicina Veterinaria, Universidad Nacional Mayor de San Marcos

2 Dirección actual: 10642 Mallard Dr. Garden Grove, CA 92843, USA

E-mail: gutierrez_romero@sbcglobal.net 
protein, the increase of digestible energy significantly enhanced WG, FC, RE, and PER, where the level of $2.7 \mathrm{kcal} / \mathrm{g}$ had the best performance. However, in diets with $35 \%$ protein, only the $2.7 \mathrm{kcal} / \mathrm{g}$ level showed good results. It can be concluded that the best response was obtained with a diet containing $2.7 \mathrm{kcal}$ of energy digestible/g and $25 \%$ protein.

Key words: digestible energy, protein, nutritional requirements, Colossoma

\section{INTRODUCCIÓN}

Los países latinoamericanos han venido fomentando la acuicultura desde hace varias décadas. En los últimos 20 años, se han sistematizado e intensificado los esfuerzos en investigación y desarrollo ante el reconocimiento de la acuicultura como fuente productora de proteína de origen animal.

Entre las especies autóctonas de la cuenca amazónica, la "gamitana" (Colossoma macropomum) ha demostrado un gran potencial para el cultivo. Como toda especie cultivable, la "gamitana" necesita asegurar la disponibilidad de alevinos para su crianza y una dieta que cubra sus requerimientos nutricionales. La proteína es uno de los más importantes nutrientes para el rendimiento piscícola, pero a su vez es uno de los componentes mas costosos en la dieta. A su vez, el nivel de energía en la dieta es crítico, pues niveles altos pueden reducir el consumo de alimento y niveles bajos demandan que se use la proteína como fuente de energía.

A nivel de América Latina, son pocos los trabajos realizados con dietas para el cultivo de peces amazónicos. Las investigaciones sobre requerimientos de proteína y energía de "gamitana" en ambientes controlados han sido limitadas y con resultados muy variados (Werder y Saint-Paul, 1978; Darmont y Salaya, 1984; Castagnolli y Zuim, 1985; Saint-Paul, 1986; Eckman, 1987; Merota y Cantelmo, 1987), llegando a recomendarse un contenido óptimo de proteína de la dieta de 23\% (Macedo, 1979; Carneiro, 1981). Sin embargo, estudios realizados en Brasil con alimento comercial para pollos conteniendo $15-17 \%$ de proteína y torta de palma hecha a partir de Orbignya martiana encontraron un crecimiento adecuado de la "gamitana" (Lovshin, 1980; Da Silva et al. 1984a). También en Brasil, se ha demostrado que crece muy bien con alimento para cerdos y carpas (CEPTA, 1987). Además, se encontró que el "pacú", Colossoma mitrei, creció adecuadamente con niveles de $25 \%$ de proteína y 2,600 Kcal de energía digestible/ $\mathrm{kg}$ cuando se utilizaron diferentes proporciones de proteína de origen animal y vegetal (CEPTA, 1987).

El objetivo del presente estudio fue desarrollar una dieta adecuadamente equilibrada en los niveles de proteína y energía, que permita lograr un efectivo desarrollo de la "gamitana".

\section{Materiales y Métodos}

El experimento se llevó a cabo en la época de vaciante (junio-octubre) en el Módulo de Nutrición de Organismos Acuáticos de la Estación Experimental de Pucallpa del Centro de Investigación IVITA de la Universidad Nacional Mayor de San Marcos, ubicada en la región de Ucayali.

Se utilizaron dieciocho tanques circulares de fibra de vidrio de $500 \mathrm{~L}$ de capacidad cada uno, sin equipamiento de aireadores ni calentadores, que estaban abastecidos con un flujo de 7.5 litros/minuto de agua constante y doblemente filtrada, la cual provenía de una represa de 15 ha de espejo de agua. La tem- 
Cuadro 1. Insumos y composición porcentual de las seis dietas experimentales utilizadas para evaluar el desarrollo de la "gamitana" (Colossoma macropomum)

\begin{tabular}{lcccccc}
\hline \multirow{2}{*}{ Insumos } & \multicolumn{7}{c}{ Dietas experimentales } \\
\cline { 2 - 7 } & 1 & 2 & 3 & 4 & 5 & 6 \\
\hline Maíz & 25.00 & 25.00 & 25.00 & 17.80 & 10.00 & 10.00 \\
Subproducto de trigo & 25.08 & 24.25 & 23.40 & 3.36 & 9.60 & 4.91 \\
Residuo de cervecería & 19.25 & 12.73 & 6.22 & 20.00 & 20.00 & 20.00 \\
Harina de pescado & 12.96 & 13.12 & 13.27 & 13.05 & 12.59 & 13.04 \\
Torta de soya & 11.22 & 15.34 & 19.47 & 41.75 & 42.00 & 42.92 \\
Aceite de pescado & 2.30 & 5.37 & 8.43 & -- & 1.74 & 5.10 \\
CO Ca & 0.69 & 0.69 & 0.91 & 0.54 & 0.57 & 0.53 \\
Vitaminas/Minerales & 0.50 & 0.50 & 0.50 & 0.50 & 0.50 & 0.50 \\
Bentonita & 3.00 & 3.00 & 3.00 & 3.00 & 3.00 & 3.00 \\
Vitamina C & 0.04 & 0.04 & 0.04 & 0.04 & 0.04 & 0.04 \\
BHT & 0.01 & 0.01 & 0.01 & 0.01 & 0.01 & 0.01 \\
Ácido propiónico & 0.05 & 0.05 & 0.05 & 0.05 & 0.05 & 0.05 \\
\hline
\end{tabular}

${ }^{1}$ Vitaminas (en UI o mg/kg de dieta): A, 5500 UI; D3, 1000 UI; E, 50 UI; K, 10; Colina, 550; Niacina, 100; Riboflavina, 20; Tiamina, 20; D-Pantetonato de Calcio, 50; Biotina, 0.10; Folacina, 5; B12, 20; Acido Ascórbico, 200; Inositol, 100. Minerales (en \% o mg/kg de dieta): Manganeso, 115; Yodo, 2.80; Cobre, 4.30; Zinc, 88; Fierro, 44; Cobalto, 0.05; Calcio, 90\%, Fósforo Disponible, 0.45\%.

peratura del agua fue de $27.0 \pm 1.4{ }^{\circ} \mathrm{C} . \mathrm{El}$ oxígeno disuelto en el agua de los estanques se mantuvo en $5.75 \pm 0.35 \mathrm{mg} / \mathrm{l}$ y el $\mathrm{pH}$ en 6.5 \pm 0.7 . Los desechos nitrogenados (alimento no comido y materia orgánica de excreción) fueron retirados diariamente.

Se utilizó una densidad de carga de 5 "gamitanas" por tanque circular, con pesos iniciales de $53.18 \pm 0.58 \mathrm{~g}$. Siete días antes del inicio del experimento, los peces fueron acostumbrados al alimento seco y pelletizado de una dieta alta en proteína y con adición de vitaminas y minerales. Además, y como medida profiláctica, los peces fueron tratados con una solución de verde de malaquita y oxitetraciclina para evitar la presencia del hongo Ichthyopthirius y bacterias patógenas. Asimismo, después de cada muestreo se em- pleó una solución de violeta de genciana o azul de metileno diluido en el agua.

Se prepararon seis dietas experimentales, que fueron formuladas por programación lineal (Programa LP88) para representar dos niveles de proteína ( 25 y $35 \%$ ) y tres niveles de energía digestible $(2.3,2.5$ y 2.7 $\mathrm{kcal} / \mathrm{g}$ ) utilizando los insumos y las cantidades indicadas en el Cuadro 1. El alimento se ofreció, ad limitum, dos veces por día (8:00 y 16:00 horas) durante 64 días. La energía digestible de las dietas fue calculada a partir de los valores calóricos de 3.5, 8.1 y $2.5 \mathrm{kcal} /$ g para proteínas, lípidos y carbohidratos respectivamente (Wilson, 1977). En el cálculo de las concentraciones de lisina, metionina y metionina + cistina se siguió los lineamientos del NRC (1983) para los insumos utilizados 
Cuadro 2. Contenido de nutrientes, energía digestible, proteína y la relación energía digestible/proteína cruda de las seis dietas experimentales utilizadas para evaluar el desarrollo de la "gamitana" (Colossoma macropomum)

\begin{tabular}{lcccccc}
\hline \multirow{2}{*}{ Nutrientes } & \multicolumn{7}{c}{ Dietas experimentales } \\
\cline { 2 - 7 } & 1 & 2 & 3 & 4 & 5 & 6 \\
\hline Lisina & 1.39 & 1.45 & 1.51 & 2.11 & 2.12 & 2.11 \\
Metionina & 0.52 & 0.52 & 0.52 & 0.66 & 0.65 & 0.65 \\
Metionina + cistina & 0.90 & 0.90 & 0.90 & 1.15 & 1.15 & 1.15 \\
Calcio & 0.90 & 0.90 & 0.90 & 0.90 & 0.90 & 0.90 \\
Fósforo disponible & 0.45 & 0.45 & 0.45 & 0.45 & 0.45 & 0.45 \\
ED ${ }^{1}$ (kcal/g) & 2.30 & 2.50 & 2.70 & 2.30 & 2.50 & 2.70 \\
Proteína (PC) & 25.00 & 25.00 & 25.00 & 35.00 & 35.00 & 35.00 \\
ED/PC & 9.20 & 10.00 & 10.80 & 6.57 & 7.14 & 7.71 \\
\hline
\end{tabular}

${ }^{1}$ La energía digestible (ED) fue calculada a partir de los valores calóricos de 3.5, 8.1 y $2.5 \mathrm{kcal} / \mathrm{g}$ para proteínas, lípidos y carbohidratos solubles, respectivamente (Wilson, 1977)

Cuadro 3. Análisis proximal (tal como ofrecido) de las seis dietas experimentales utilizadas para evaluar el desarrollo de la "gamitana" (Colossoma macropomum)

\begin{tabular}{lcccccc}
\hline \multirow{2}{*}{ Parámetro } & \multicolumn{7}{c}{ Dietas experimentales } \\
\cline { 2 - 7 } & 1 & 2 & 3 & 4 & 5 & 6 \\
\hline Humedad & 11.75 & 11.49 & 11.12 & 11.89 & 11.89 & 11.50 \\
Proteína & 23.85 & 23.87 & 23.76 & 31.93 & 32.11 & 31.92 \\
Lípidos & 6.80 & 10.53 & 13.00 & 4.53 & 6.53 & 9.07 \\
Fibra & 9.33 & 8.10 & 5.67 & 9.00 & 8.53 & 8.33 \\
Ceniza & 8.10 & 7.75 & 8.13 & 8.95 & 8.90 & 7.95 \\
Extracto LN & 40.17 & 38.26 & 38.32 & 33.70 & 32.04 & 31.20 \\
\hline
\end{tabular}

(Cuadro 2). La formulación de las concentraciones de lisina, metionina y metionina + cistina en las dietas se hicieron en forma similar al contenido de estos aminoácidos en la carcasa de la "gamitana", debido a que se ha encontrado una estrecha relación entre el patrón de requerimientos de aminoácidos esenciales de los peces y el patrón de aminoácidos esenciales de la proteína corporal (Nose, 1979; Mambrini y Kaushik, 1995).

Se usó el análisis químico proximal (AOAC, 1990) de los insumos utilizados en las dietas para determinar los contenidos de humedad, proteína, lípidos, fibra, ceniza y extracto libre de nitrógeno (Cuadro 3). La 
Cuadro 4. Ganancia de peso, conversión alimenticia, energía retenida, y relación de eficiencia proteica de alevinos de gamitana (Colossoma macropomum) alimentados durante 9 semanas con 6 dietas experimentales que representaron dos niveles de proteína $(25 \%$ y $35 \%$ ) y tres niveles de energía digestible $(2.3,2.5$ y $2.7 \mathrm{kcal} / \mathrm{g})$

\begin{tabular}{|c|c|c|c|c|c|c|}
\hline & \multicolumn{3}{|c|}{$25 \%$} & \multicolumn{3}{|c|}{$35 \%$} \\
\hline & 2.3 & 2.5 & 2.7 & 2.3 & 2.5 & 2.7 \\
\hline Ganancia de peso ${ }^{1}(\%)$ & $64.2 \pm 4.9^{\mathrm{a}}$ & $65.7 \pm 2.7^{\mathrm{a}}$ & $81.8 \pm 0.1^{\mathrm{c}}$ & $59.7 \pm 8.7^{\mathrm{b}}$ & $59.5 \pm 4.9^{\mathrm{b}}$ & $73.4 \pm 2.2^{\mathrm{d}}$ \\
\hline Conversión alimenticia ${ }^{2}$ & $1.89 \pm 0.05^{\mathrm{a}}$ & $1.84 \pm 0.05^{\mathrm{a}}$ & $1.51 \pm 0.02^{\mathrm{b}}$ & $1.98 \pm 0.11^{\mathrm{c}}$ & $2.01 \pm 0.14^{d}$ & $1.66 \pm 0.04^{\mathrm{e}}$ \\
\hline Energía retenida $^{3}$ & $19.8 \pm 0.26^{\mathrm{a}}$ & $21.04 \pm 0.45^{\mathrm{b}}$ & $29.3 \pm 0.13^{\mathrm{c}}$ & $25.9 \pm 1.02^{\mathrm{d}}$ & $20.5 \pm 1.11^{\mathrm{a}}$ & $25.9 \pm 0.48^{\mathrm{d}}$ \\
\hline $\begin{array}{l}\text { Relación de eficiencia } \\
\text { proteica }^{4}\end{array}$ & $2.12 \pm 0.06^{\mathrm{a}}$ & $2.17 \pm 0.05^{\mathrm{a}}$ & $2.64 \pm 0.03^{\mathrm{b}}$ & $1.44 \pm 0.08^{\mathrm{c}}$ & $1.42 \pm 0.10^{c}$ & $1.72 \pm 0.04^{\mathrm{d}}$ \\
\hline
\end{tabular}

$a, b, c, d, e$ Letras diferentes dentro de filas indican diferencias significativas $(p<0.05)$

1 (Peso Final-Peso Inicial)/Peso Inicial x 100

2 Ingesta de alimento $(\mathrm{g}) /$ ganancia de peso $(\mathrm{g})$

3 (Energía corporal final - Energía corporal inicial)/ingesta de ED x 100

${ }^{4}$ Ganancia de peso (g) / Proteína cruda consumida (g)

composición química de la carcasa (proteínas, lípidos, cenizas y humedad expresados en base húmeda), se determinó al inicio y al final del experimento sobre una muestra de seis peces por tratamiento (AOAC, 1990). Los datos se utilizaron para calcular la energía retenida.

El comportamiento productivo de la "gamitana" se evaluó a través de la ganancia de peso (Hopkins, 1992), conversión alimenticia, energía retenida (Reinitz y Hitzel, 1980), y la eficiencia proteica (Hepher, 1993).

Se empleó un diseño factorial de $2 \times 3$, con seis tratamientos y tres repeticiones por tratamiento. Los valores obtenidos fueron sometidos al análisis de variancia, y las diferencias entre promedios se determinaron con la Prueba de Rangos Múltiples de Duncan, empleando el paquete estadístico Statigraphics v 5.1 (1991).

\section{Resultados y Discusión}

A medida que la energía digestible se incrementó en las dietas con $25 \%$ de proteína se incrementó la ganancia de peso $(\mathrm{p}<0.05)$; sin embargo, solo el nivel más alto de energía digestible afectó significativamente el crecimiento en las dietas con $35 \%$ de proteína. Así mismo, para igual nivel de energía digestible, la ganancia de peso fue más alta con el nivel más bajo de proteína (Cuadro 4). Los resultados indican que la mayor ganancia de peso se obtuvo con la dieta de $2.7 \mathrm{kcal} /$ g y $25 \%$ de proteína. En otros estudios y en similares condiciones se encontró una mejor respuesta en el crecimiento de esta especie utilizando dietas isocalóricas $(2.85 \mathrm{kcal} / \mathrm{g})$ y con una alta proporción de proteína animal (Eckman, 1987). Por otro lado, en alimentación de gamitanas con dietas isocalóricas (2.8 $\mathrm{kcal} / \mathrm{g}$ )) con 0,25 y $95 \%$ de proteína animal, se encontró que la dieta intermedia expresó 
una mejor respuesta en términos de crecimiento (Werder y Saint Paul, 1978). Resultados similares fueron obtenidos en gamitana (Gutiérrez et al., 1996a), Colossoma mitrei (Carneiro et al., 1984b) y en "paco" Piaractus brachypomus (Gutiérrez et al., 1996b).

Se encontraron diferencias altamente significativas $(\mathrm{p}<0.01)$ entre los niveles de energía digestible con relación a conversión alimenticia (Cuadro 4). Para ambos niveles de proteína (25 y 35\%), conforme se incrementó el nivel de energía digestible, mejoró la conversión alimenticia. No se encontró diferencia significativa entre niveles de proteína. Estadísticamente, la mejor conversión alimenticia se encontró con la dieta de $2.7 \mathrm{kcal} / \mathrm{g}$ y $25 \%$ de proteína. Sin embargo, los niveles obtenidos en el presente estudio son más bajos que en otros estudios; así, Werder y Saint-Paul (1978) obtuvieron una conversión de 2.3 con una dieta de $30 \%$ de proteína cruda, Da Silva et al. (1978) obtuvo 2.8 con $27 \%$ de proteína, y Lovshin et al. (1974) de 3.3 con $29.1 \%$ de proteína.

En el nivel de $25 \%$ de proteína en la dieta (Cuadro 4), a medida que se incrementó el nivel de energía digestible se incrementó el porcentaje de energía retenida $(\mathrm{p}<0.05)$; así mismo, el análisis factorial demostró que la mejor respuesta, en términos de energía retenida se encontró con la dieta de $2.7 \mathrm{kcal} / \mathrm{g}$ y $25 \%$ de proteína. No se encontró información de energía retenida para la especie en estudio; sin embargo, se dispone de un estudio donde se evaluó un modelo empírico para calcular la energía digestible requerida por la "trucha arco iris" Oncorhynchus mykiss, donde se demostró que se requiere $3.59 \mathrm{kcal}$ de ED para producir $1.91 \mathrm{kcal}$ de energía retenida en base seca (Cho y Kaushik, 1990), en tanto que en el presente estudio se requirió $2.7 \mathrm{kcal}$ de ED para producir $1.13 \mathrm{kcal}$ de energía retenida en base seca.

La menor energía digestible usada por la gamitana puede explicarse por la diferencia en los hábitos alimenticios, composición de la dieta, tasa de alimentación y composición de la ganancia corporal, factores que afectan significativamente el balance energético de los peces. Lucket y Moreau (1989) sostienen que el rápido crecimiento de los peces de aguas tropicales se explica por la habilidad que tienen para usar mejor la proteína suministrada. Esto no es debido solo a una mejor capacidad de retención de energía proteica, sino también a la mejor utilización de la energía no proteica, ahorrando la proteína para propósitos de síntesis de tejidos. En términos de retención de energía, la dieta con $25 \%$ de proteína y $2.7 \mathrm{kcal}$ de $\mathrm{ED} / \mathrm{g}$ de alimento fue la mejor, posibilitando una mayor incorporación de los insumos energéticos (maíz amarillo duro y aceite de pescado) como fuentes de energía, ahorrando la proteína para el crecimiento (Kaushik y Cowey, 1991; Kaushik y Medale, 1994; Medale et al., 1995).

El análisis de los resultados demostró diferencias altamente significativas $(p<0.01)$ entre los niveles de energía digestible, proteína en la dieta y la interacción energía digestible-proteína. La relación de eficiencia proteica (REP) se incrementó conforme aumentó el nivel de energía digestible con dietas de $25 \%$ de proteína, mas no así con 35\% de proteína (Cuadro 4). El análisis estadístico indicó que la dieta de $2.7 \mathrm{kcal}$ de $\mathrm{ED} / \mathrm{g}$ y $25 \%$ de proteína fue la más eficiente en términos de la REP. La literatura indica que el máximo crecimiento de "tilapia híbrida" se obtuvo con una dieta de $24 \%$ de proteína y una REP de 2.99 (Shiau y Ling Huang, 1989, 1990). Asimismo, con dietas de $20 \%$ de proteína en "tilapia roja" se obtuvo una REP de 2.41, lográndose una mejor eficiencia de utilización de la proteína, al compararse con dietas de mayor nivel de proteína (Clark et al., 1990). Datos similares han sido obtenidos en otros estudios con "carpa cabezona" Aristichthys nobilis (Santiago y Reyes, 1991) y en otras especies de tilapia (Teshima et al., 1978; Mazid et al., 1979; Jauncey, 1982; Teshima et al., 1985; Siddiqui et al., 1988). No obstante, El Sayed y Teshima (1992) encontraron para "tilapia del nilo" 
Oreochromis niloticus el mejor valor de la REP en una dieta de $45 \%$ de proteína y 3.0 kcal de energía bruta/g.

La literatura muestra un amplio rango de variación en relación a la concentración de proteína en la dieta de la gamitana o peces de similares hábitos alimenticios como la tilapia, fluctuando entre 20 y $45 \%$. Sin embargo, son escasos los datos sobre los niveles de energía digestible necesarios para un adecuado crecimiento de la gamitana. Los resultados encontrados en el presente estudio indican que el incremento del nivel de energía digestible, independiente del nivel de proteína usado, mejoró significativamente la ganancia de peso, conversión alimenticia, energía retenida y la eficiencia proteica. Además, se encontró que $2.7 \mathrm{kcal}$ de $\mathrm{ED} / \mathrm{g}$ es el mejor nivel energético; por lo que se puede concluir, considerando las condiciones del experimento y el costo de la proteína, que la mejor respuesta animal se puede obtener con la dieta de $25 \%$ de proteína y $2.7 \mathrm{kcal}$ de $\mathrm{ED} / \mathrm{g}$.

\section{Literatura Cittada}

1. Andrews JW, Page JW. 1974. Growth factors in fish meal components of catfish diets. J Nutr. 104: 1091-1096.

2. [AOAC] Association of Official Analytical Chemist. 1990. Official methods of analysis, $15^{\text {th }}$ ed. Virginia, USA: AOAC, $957 \mathrm{p}$.

3. Carneiro DJ. 1981. Digestibilidade proteica em dietas isocalóricas para o tambaquí, Colossoma macropomum (Cuvier, Pises). II Simposio Bras Aquicultura. Brasilia: Encontro Nac. Ranicult./SUDEPE. p 788-800.

4. Carneiro DJ, Castagnolli N, Machado CR, Verardino M. 1984b. Nutricio do pacú, Colossoma mitrei (Berg, 1895). III Niveis de energia metabolizavel em dietas isoproteicas. São Carlos, SP: Simpósio Brasileiro de Acuicultura. $\mathrm{p}$ 133-146.
5. Castagnolli N, Zuim SMF. 1985. Consolidacao do conhecimento adquirido sobre o pacú (Colossoma mitrei Berg 1895). Bol Tec Jabocatibal, FCAU. 30 p.

6. [CEPTA] Centro de Pesquisa e Treinamento em Acuicultura. 1977. Sintese dos trabalhos realizados com espéces do genero Colossoma. Pirassununga, Sao Paulo. 37 p.

7. Cho CY, Kaushik SJ. 1990. Nutritional energetic in fish: energy and protein utilization in rainbow trout Salmo gairdneri. World Rev Nutr Dict 61: 132172.

8. Clark AE, Watanabe WO, Olla BL, Wicklund RI. 1990. Growth, feed conversion and protein utilization of Florida red tilapia fed isocaloric diets with different protein levels in seawater pools. Aquaculture 88: 75-85.

9. Da Silva AB, Carneiro D, Sobrinho F, Melo R. 1978. Monocultivo del "tambaqui" Colosoma macropomum. CERLA, Brasil. $32 \mathrm{p}$.

10. Da Silva AB, Lovshin LL, Dos Santos EP, De Mello JTC, De Mello FR, Sobrinho A. 1984a. Analise complementar de um ensaio em piscicultura intensiva de pirapitinga, Colossoma macropomum. Cienc Cult 36: 436-438.

11. Darmont M, Salaya JJ. 1984. Ensayo de cultivo de la cachama Colossoma macropomum Cuvier 1818, en jaulas flotantes rígidas. Mem Asoc Latinoam Acuicult 5: 465-479.

12. Eckman R. 1987. Growth and body composition of juvenile Colossoma macropomum Cuvier, 1818 (Characoidei) feeding on artificial diets. Aquaculture 64: 293-303.

13. El-Sayed AF, Teshima S.1992. Protein and energy requirements of nile tilapia Oreochromis niloticus fry. Aquaculture 103: 55-63.

14. Fukushima D. 1968. Internal structure of $7 \mathrm{~s}$ and $11 \mathrm{~s}$ globulin molecules in soybean protein. Cereal Chem 45: 203-224.

15. Gutiérrez AW, Zaldívar J, Rebaza J. 1996a. Utilización de dietas prácticas con diferentes niveles de aminoácidos 
azufrados totales para el crecimiento de gamitana (Colossoma macropomum), Pisces Characidae. Folia Amazónica 7(12): 195-200.

16. Gutiérrez W, Zaldívar J, Deza S, Rebaza M. 1996b. Determinación de los requerimientos de proteína y energía de juveniles de "paco" (Piaractus brachypomus), Pisces Characidae. Folia Amazónica 8(2): 35-45.

17. Hepher B. 1993. Nutrición de peces comerciales en estanques. México: Ed. Limusa. $406 \mathrm{p}$.

18. Hopkins KD. 1992. Reporting fish growth: A review of the basis. J World Aquaculture Soc 23(3): 173-179.

19. Jauncey K. 1982. The effects of varying dietary protein level on the growth, food conversion, protein utilization and body composition of juvenile tilapias (Sarotherodon mossambicus). Aquaculture 27: 43-54.

20. Kaushik SJ, Cowey CB. 1991. Ammoniogenesis and dietary factors affecting nitrogen excretion. In: Cowey $\mathrm{CB}$, Cho CY, eds. Nutritional strategies and aquaculture waste. Guelph, Canada: Univ. Guelph. p 3-19.

21. Kaushik SJ, Medale F. 1994. Energy requirements utilization and supply to salmonids. Aquaculture 124: 81-97.

22. Lovhin LL, Da Silva AB, Fernandez J, Carneiro A. 1974. Preliminare pond cultura test of "pirapitinga" Colossoma bidens and "tamabaqui" Colossoma macropomum for the amazonriver basin. FAO. Informe de Pesca $\mathrm{N}^{\mathrm{o}} 159$. Vol I. 8 p.

23. Lovshin LL. 1980. Situación del cultivo de Colossoma sp. en Sud América. Rev Latinoam Acuicult. 5: 27-42.

24. Luquet P, Moreau Y. 1989. Energyprotein management by some warm water finfish. In: Advances in tropical aquaculture workshop at Tahiti, French Polynesia. Acts of Colleges No 9. p 751756.

25. Macedo EM. 1979. Necessidade proteica na nutrica do tambaqui Colossoma macropomum Cuvier 1818 (Pises Characidae). M.Sc. Thesis.
Jabocatibal, Brasil: Univ. Estadual Paulista. $71 \mathrm{p}$.

26. Mambrini M, Kaushik SJ.1995. Indispensable amino acids requirements of fish: Correspondence between quantitative data and amino acids profile of tissue protein. J Appl Ichthyol 11: 240-247.

27. Mazid MA, Tanaka Y, Katayama T, Rahman MA, Simpson KL, Chichester CO. 1979. Growth response of Tilapia zilli fingerling fed isocaloric diets with variable protein levels. Aquaculture 18: 115-122.

28. Medale FC, Brauge C, Vallée F, Kaushik SJ. 1995. Effects of dietary protein/energy ratio, ration size, dietary energy source and temperature on nitrogen excretion in rainbow trout. Water Sci Technol 31(10): 185-195.

29. Merota N, Cantelmo OA. 1987. Growth, feed conversion, and mortality of cage-reared Tambaqui, Colossoma macropomum, fed various dietary feeding regimes and protein levels. Aquaculture 66: 223-233.

30. [NRC] National Research Council. 1983. Nutrient requirements of warm water fishes and shellfishes. Revised ed. Washington. DC, USA: National Academy Press.102 p.

31. Nose T. 1979. Summary report on the requirements of essential amino acids for carp. In: Halver JE, Tiews K, eds. Finfish nutrition and fish feed technology. Berlin: Heenemann. p 145-156.

32. Saint-Paul U. 1984a. Ecological and fisiological investigation of Colossoma macropomum, a new species for fish culture in Amazonia. Mem Asoc Latinoam Acuilt 5: 501-518.

33. Saint-Paul U. 1986. Potential for aquaculture of South American freshwater fishes: a review. Aquaculture 54: 205-240.

34. Santiago CB, Reyes OS. 1991. Optimum dietary protein level for growth of bighead carp Aristichthys nobilis fry in a static water system. Aquaculture 93: 155-162. 
35. Shiau SY, Huang SL. 1989. Optimal dietary level for hybrid tilapia (Oreochromis niloticus $x$ O. aureus reared is seawater. Aquaculture 81: 119127.

36. Shiau SY, Huang SL. 1990. Influence of varying energy levels with two protein concentrations in diets for hybrid tilapia (Oreochromis niloticus $x$ O.aureus) reared in seawater. Aquaculture 82: 110117.

37. Siddiqui AQ, Howlander MS, Adam $\boldsymbol{A A}$. 1988. Effects of dietary protein levels on growth, feed conversion and protein utilization in fry and young nile tilapia Oreochromis niloticus. Aquaculture 70: 63-73.

38. Smith RR, Winfree RA, Rumsey GW, Allred A, Peterson M. 1995. Apparent digestion coefficients and metabolizable energy of feed ingredients for rainbow trout Oncorhynchus mykiss. J World Aquacult Soc 26(4): 432-437.
39. Statigraphics. 1991. Statical graphics system. Statical Graphics Corporation, MD, USA.

40. Teshima S, Gonzales GMO, Kanazawa A. 1978. Nutritional requirements of tilapia: utilization of dietary by Tilapia zilli. Mem Fac Fish, Kagoshima Univ. 27: 49-57.

41. Teshima S, Kanazawa A, Uchiyama Y. 1985. Optimum protein levels in casein-gelatin diets for Tilapia niloticus fingerling. Mem Fac Fish, Kagoshima Univ. 34: 45-52.

42. Werder U, Saint-Paul U. 1978. Feeding trials with herbivorous and omnivorous Amazonian fishes. Aquaculture 15: 175-177.

43. Wilson RP. 1977. Energy relationships in catfish diets. In: Stickney RR, Lovell RT, eds. Nutrition and feeding of channel catfish. Southern Cooperative Series. Bull. 218: 21-29. 